\title{
The Role of Dynamic Subtraction MRI in Assessment of Reactivity of HFLS Post TACE by 3 Tesla MRI Machine
}

\author{
Medhat M. Reffat, Wael Elshawaf, Shorouk Z. Abdel Shafy, Khaled E. Aly
}

Department of Radiology, Benha faculty of medicine, Benha University, Egypt

Correspondence to: Khaled E. Aly, Department of Radiology, Benha faculty of medicine, Benha University, Egypt

Email:

khalidelsaied2020@gmail.com

Received:

Accepted

\begin{abstract}
:
Background: Hepatocellular carcinoma (HCC) is one of the most common cancers worldwide, and has a poor prognosis unless treated. The aim of this work was to assess HCC cases after transarterial chemoembolization by subtraction dynamic contrast enhanced MRI to detect its accuracy, sensitivity and specificity in detecting residual tumor and assess the need for further treatment. Methods: 35 patients with $42 \mathrm{HCC}$ lesions underwent transarterial chemoembolization were included in this study. Those patients underwent MRI after 3 months where dynamic MRI and subtraction dynamic MRI were obtained. The interpretation of dynamic MRI and the subtraction dynamic MRI was done by two different readers experienced in hepatic imaging blinded to each other. Patients were classified according to reactivity rate into 2 independent groups: Reactive group (30 patients), Not reactive group (5 patients). Results: Comparative study between D-MRI
\end{abstract} and DS-MRI assessments revealed; highly significant increase in reactivity rate in DS-MRI in HCC patients during $1^{\text {st }}$ and $2^{\text {nd }}$ readings; with highly significant difference $(\mathrm{p}=0.0078$ respectively). By using ROC-curve analysis, DS-MRI assessment discriminated patients with reactivity from patients without, with perfect accuracy, sensitivity $=100 \%$ and specificity $=100 \%$ ( $\mathrm{p}<0.0001)$. Conclusion: Dynamic MRI is valuable in detecting recurrent lesions however, this value is augmented by the addition of subtraction technique especially in lesions having high signal before administration of contrast medium.

Keywords: Dynamic Subtraction; MRI; HCC; post TACE; 3 tesla 


\section{Introduction}

Hepatocellular carcinoma (HCC) is a primary malignancy of the liver and occurs predominantly in patients with underlying chronic liver disease and cirrhosis. HCC is now the third leading cause of cancer deaths worldwide, with over 500,000 people affected (1).

Transplantation remains the best option for patients with HCC. Unfortunately, there is a limited supply of good-quality deceased donor organs. Thus, alternative treatments, including resection, radiofrequency ablation (RFA), and, potentially, systemic therapy (2).

Patients who have advanced disease may benefit from palliative care interventions rather than be subjected to often ineffective therapies (3).

The most commonly offered therapy is transcatheter arterial chemoembolization (TACE). TACE is performed by an interventional radiologist who selectively cannulates the feeding artery to the tumor and delivers high local doses of chemotherapy, including doxorubicin, cisplatin, or mitomycin C. To prevent systemic toxicity, the feeding artery is occluded with gel foam or coils to prevent flow (4).
Unenhanced CT confirms successful introduction of the chemoembolization mixture into the targeted lesions. However, it can be difficult to evaluate contrast enhancement in a tumor with partial retention of iodized oil on contrastenhanced CT because of the beam hardening artifacts produced by the high attenuation of iodized oil. The signal intensity of MRI is not degraded by the presence of iodized oil; therefore, a residual viable tumor is better defined by MRI (5). Dynamic contrast-enhanced MRI (DCE MRI) can play a significant role as an imaging biomarker, especially in the detection of viable tumor foci, the differentiation between necrosis and viable tumor, and early prediction of response (6). Subtraction imaging is a technique where a non-enhanced T1-weighted sequence is digitally subtracted from the T1-weighted image achieved after gadolinium administration. Thus the subtraction imaging is helpful for the assessment of the therapeutic efficacy for post TACE HCCs, which makes the detection of tumor enhancement difficult on post-contrast T1weighted images. By accomplishing this operation, any native T1 signal is removed 
and the remaining signal on the subtracted images is only due to enhancement (7).

The aim of this work was to assess HCC cases after trans-arterial chemoembolization by subtraction dynamic contrast enhanced MRI to detect its accuracy, sensitivity and specificity in detecting residual tumor and assess the need for further treatment.

\section{Patients and methods}

This was a retrospective comparative study was conducted on 35 patients hepatocellular carcinoma (HCC); to assess HCC cases after trans-arterial chemoembolization (TACE) by subtraction dynamic contrast enhanced MRI to detect its accuracy, sensitivity and specificity in detecting residual tumor and assess the need for further treatment. This study was conducted on patients with HCC who underwent TACE from January 2017 till December 2019 at Maadi Military Hospital. Patients were referred from hepatology department of Maadi Military hospital. This study was approved by the local institutional ethical committee.

Patients included in our study were selected with the following criteria:

- Age group: 50-80

- Both sexes are included.
- Known patient with HCC who underwent TACE and accepted to undergo post contrast dynamic MRI examination.

\section{The following patients were excluded} from the study:

- Patients with contraindications to contrast media, e.g. patients with renal failure and patients allergic to contrast media.

- Patients with contraindications to magnetic resonance imaging, e.g. claustrophobia, cardiac prosthesis and metallic plates

- Patients who underwent procedures other than TACE e.g. TARE and RFA.

- Patients refused to do the study.

The patients were subjected to:

- Consent taking.

- Clinical assessment.

- Checking for contraindication to MRI imaging (e.g. pacemaker, metallic implant, and severe claustrophobia).

- Revision of the patient's laboratory investigations including renal function tests (mainly serum creatinine).

- Revision of the radiological investigations previously done for the patients.

- Patients underwent dynamic MRI with DWI and Subtraction sequences within 
90 days after one or more sessions of TACE.

\section{Statistical Analysis:}

Data entry, processing and statistical analysis was carried out using MedCalc ver. 15.8. (MedCalc, Ostend, Belgium). Tests of significance (Mann-Whitney's, McNemar's, Chi square tests, Kappa statistics, and ROC Curve analysis) were used. Data were presented and suitable analysis was done according to the type of data (parametric and non-parametric) obtained for each variable. P-values less than $0.05(5 \%)$ was considered to be statistically significant. Descriptive statistics: Mean, Standard deviation ( \pm SD) and range for parametric numerical data, while Median and Inter-quartile range (IQR) for non-parametric numerical data and Frequency and percentage of nonnumerical data. Analytical statistics:_MannWhitney's Test (U test) was used to assess the statistical significance of the difference of a non-parametric variable between two study groups. Chi-Square test was used to examine the relationship between two qualitative variables. The ROC Curve (receiver operating characteristic) provides a useful way to evaluate the Sensitivity and specificity for quantitative Diagnostic measures that categorize cases into one of two groups.

\section{Results}

The mean age of all patients was $(62.31 \pm$ 7.14) years. Regarding gender of the patients, the majority (88.6\%) of patients were males; while only $(11.4 \%)$ were females.

Regarding D-MRI data, the average number and size of lesions were (1.22 \pm $0.49),\left(13.13 \pm 9.91 \mathrm{~cm}^{3}\right)$ respectively, in $1^{\text {st }}$ and $2^{\text {nd }}$ readings. Regarding reactivity rate in D-MRI, (62.9\%) of patients were reactive in $1^{\text {st }}$ and $2^{\text {nd }}$ readings. However, signal restriction rate in $1^{\text {st }}$ reading was $(42.9 \%)$, and in $2^{\text {nd }}$ reading was $(45.7 \%)$. Regarding reactivity rate in DS-MRI, $(85.7 \%)$ of patients were reactive in $1^{\text {st }}$ and $2^{\text {nd }}$ readings. Table 1.

The 35 HCC patients were classified according to reactivity rate into 2 independent groups: Reactive group (30 patients) and Not reactive group (5 patients). Comparative study between the 2 groups revealed non-significant difference as number and size of lesions during $1^{\text {st }}$ reading $(\mathrm{p}>0.05)$. Comparative study between the 2 groups revealed; highly significant increase in reactivity rate of ( $\mathrm{D}$ and DS-MRI) during $1^{\text {st }}$ reading; in 
reactive group compared to non-reactive group; with highly significant statistical difference $(\mathrm{p}<0.01$ respectively). Comparative study between the 2 groups revealed non-significant difference as regards signal restriction rate of (D-MRI) during $1^{\text {st }}$ reading $(\mathrm{p}>0.05)$. Table 2 .

Comparative study between the 2 groups revealed non-significant difference as number and size of lesions during $2^{\text {nd }}$ reading $(\mathrm{p}>0.05)$. Comparative study between the 2 groups revealed; highly significant increase in reactivity rate of (D and DS-MRI) during $2^{\text {nd }}$ reading; in reactive group compared to non-reactive group; with highly significant statistical difference $(\mathrm{p}<0.01$ respectively). Comparative study between the 2 groups revealed non-significant difference as regards signal restriction rate of (D-MRI) during $2^{\text {nd }}$ reading $(\mathrm{p}>0.05)$. Table 3
We further analyzed and compared al the 35 (paired) HCC patients according to the serial D and DS-MRI assessments. Comparative study between D-MRI and DS-MRI assessments revealed; highly significant increase in reactivity rate in DSMRI in HCC patients during $1^{\text {st }}$ and $2^{\text {nd }}$ readings; with highly significant difference ( $p=0.0078$ respectively). Figure 1

By using ROC-curve analysis, D-MRI assessment discriminated patients with reactivity from patients without, with good accuracy, sensitivity $=73 \%$ and specificity= $100 \%$ ( $\mathrm{p}<0.0001)$. By using ROC-curve analysis, DS-MRI assessment discriminated patients with reactivity from patients without, with perfect accuracy, sensitivity= $100 \%$ and specificity $=100 \%(\mathrm{p}<0.0001)$. Figure 2

Table (1): MRI data among $35 \mathrm{HCC}$ patients

\begin{tabular}{|c|c|c|c|}
\hline Variables & & (Reader-1) & (Reader-2) \\
\hline \multirow{4}{*}{ D-MRI } & Number of lesions & $1.22 \pm 0.49$ & $1.22 \pm 0.49$ \\
\hline & Size of lesions $\left(\mathrm{cm}^{3}\right)$ & $13.13 \pm 9.91$ & $13.13 \pm 9.91$ \\
\hline & Reactivity rate (D-MRI) & $22(62.9 \%)$ & $22(62.9 \%)$ \\
\hline & Signal restriction rate & $15(42.9 \%)$ & $16(45.7 \%)$ \\
\hline DS-MRI & Reactivity rate (DS-MRI) & $30(85.7 \%)$ & $30(85.7 \%)$ \\
\hline
\end{tabular}

D-MRI: dynamic MRI. DS-MRI: digital subtraction MRI. 
Table (2): Comparison between the 2 groups as regards MRI data $\left(1^{\text {st }}\right.$ reading) using Mann-Whitney's $U$ and Chi square tests.

\begin{tabular}{|c|c|c|c|c|}
\hline \multicolumn{2}{|l|}{ Variable } & $\begin{array}{l}\text { Non-reactive group (5) } \\
\text { Median (IQR) }\end{array}$ & $\begin{array}{l}\text { Reactive group (30) } \\
\text { Median (IQR) }\end{array}$ & $\begin{array}{l}\text { Mann-Whitney's U test } \\
\text { P value }\end{array}$ \\
\hline \multirow{2}{*}{\multicolumn{2}{|c|}{$\begin{array}{l}\text { Number of lesions } \\
\text { Size of lesions }\left(\mathrm{mm}^{3}\right)\end{array}$}} & $1(1-1.5)$ & $1(1-1)$ & $=0.838$ \\
\hline & & $10.5(6.6-16.5)$ & $10.2(6.2-18)$ & $=0.981$ \\
\hline \multicolumn{2}{|l|}{ Variable } & $\begin{array}{l}\text { Non-reactive group } \\
\text { (5) }\end{array}$ & $\begin{array}{l}\text { Reactive group } \\
\text { (30) }\end{array}$ & $\begin{array}{l}\text { Chi square test } \\
\text { P value }\end{array}$ \\
\hline \multirow{2}{*}{ D-MRI } & $\begin{array}{l}\text { Reactivity rate (D- } \\
\text { MRI) }\end{array}$ & $\mathbf{0}(\mathbf{0 \%})$ & $22(73.3 \%)$ & $=0.008 * *$ \\
\hline & $\begin{array}{l}\text { Signal restriction } \\
\text { rate }\end{array}$ & $\mathbf{0}(\mathbf{0 \%})$ & $15(50 \%)$ & $=0.108$ \\
\hline DS-MRI & $\begin{array}{l}\text { Reactivity rate } \\
\text { (DS-MRI) }\end{array}$ & $\mathbf{0}(\mathbf{0 \%})$ & $30(100 \%)$ & $<0.0001 * *$ \\
\hline
\end{tabular}

* Percentage of Column Total.

Table (3): Comparison between the 2 groups as regards MRI data ( $2^{\text {nd }}$ reading) using Mann-Whitney's U and Chi square tests:

\begin{tabular}{|c|c|c|c|c|}
\hline \multicolumn{2}{|l|}{ Variable } & $\begin{array}{l}\text { Non-reactive group (5) } \\
\text { Median (IQR) }\end{array}$ & $\begin{array}{l}\text { Reactive group (30) } \\
\text { Median (IQR) }\end{array}$ & $\begin{array}{l}\text { Mann-Whitney's } \\
\text { U test } \\
\text { P value }\end{array}$ \\
\hline \multirow{2}{*}{\multicolumn{2}{|c|}{$\begin{array}{l}\text { Number of lesions } \\
\text { Size of lesions }\left(\mathrm{mm}^{3}\right)\end{array}$}} & $1(1-1.5)$ & $1(1-1)$ & $=0.838$ \\
\hline & & $10.5(6.6-16.5)$ & $10.2(6.2-18)$ & $=0.981$ \\
\hline \multicolumn{2}{|l|}{ Variable } & $\begin{array}{l}\text { Non-reactive group } \\
\text { (5) }\end{array}$ & $\begin{array}{l}\text { Reactive group } \\
\text { (30) }\end{array}$ & Chi square test \\
\hline \multirow{2}{*}{ D-MRI } & Reactivity rate (D-MRI) & $0(0 \%)$ & $22(73.3 \%)$ & $=0.008 * *$ \\
\hline & Signal restriction rate & $0(0 \%)$ & $16(53.3 \%)$ & $=0.083$ \\
\hline DS-MRI & Reactivity rate (DS-MRI) & $0(0 \%)$ & $30(100 \%)$ & $<0.0001 * *$ \\
\hline
\end{tabular}

* Percentage of Column Total.

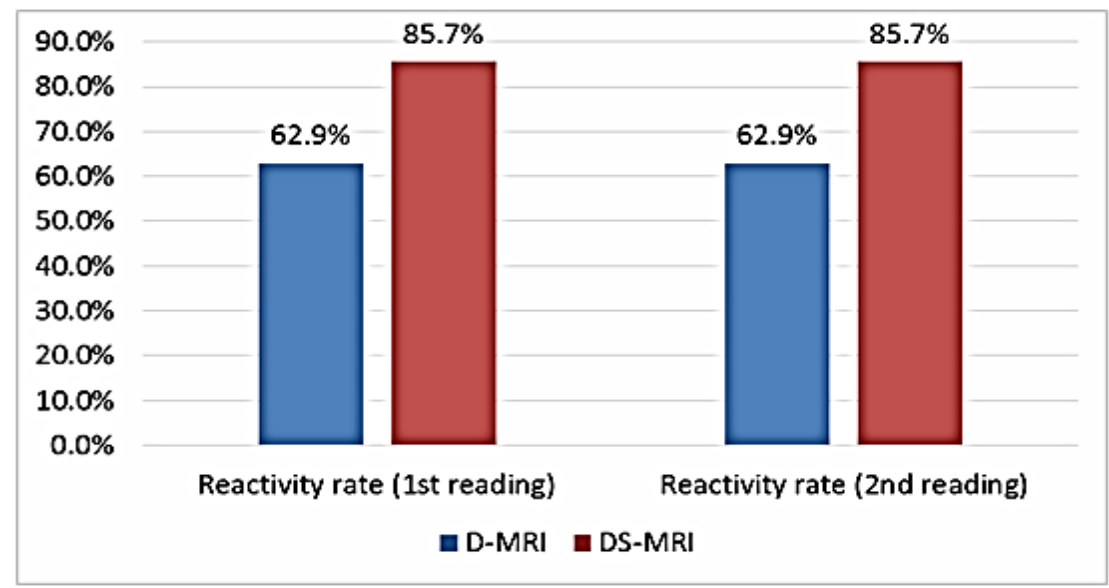

Figure (1): Comparison D-MRI and DS-MRI reactivity assessments. 


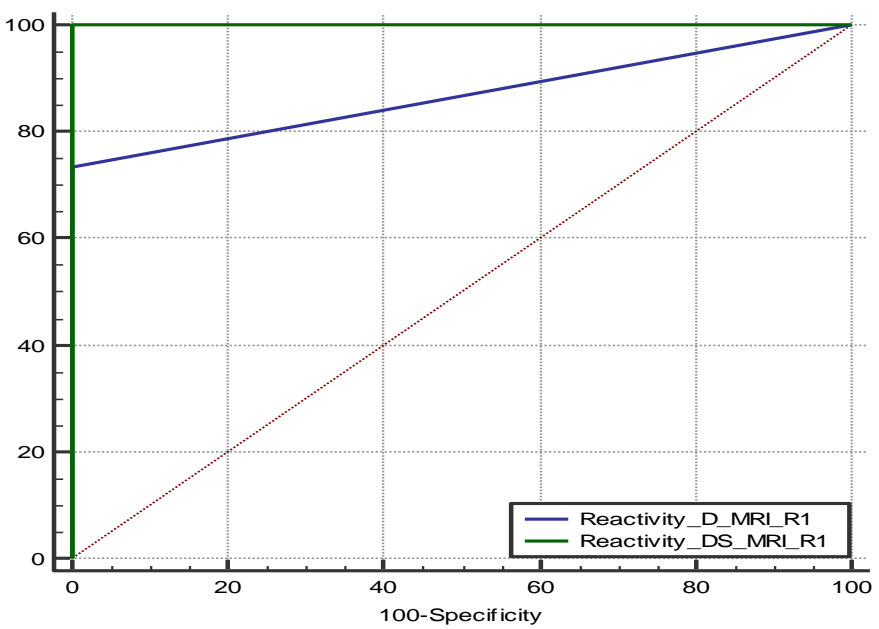

Figure (2): ROC curve of D-MRI vs DS-MRI.

\section{Discussion}

The aim of this study was to evaluate the role of Subtraction Dynamic MRI in the detection of recurrent or residual tumor viability after transarterial chemoembolization.

Our results agreed with study done at (8) which showed that contrast enhanced MRI with subtraction technique revealed a significant correlation with the histopathological findings more than DWI, in the evaluation of necrosis in $\mathrm{HCC}$ following TACE.

Our study also agreed with study done at (9) which stated that DWI was not a reliable predictor of local HCC recurrence following TACE compared to gadolinium-enhanced MRI.
Our results were different from those elicited by study done at (10), which observed that diffusion weighted imaging had a $100 \%$ sensitivity, a $65.5 \%$ specificity, a positive predictive value of $67.7 \%$, a negative predictive value of $100 \%$ and an overall agreement of $80 \%$.

Our results correlated with study done at (11) which reported a sensitivity of $92 \%$, specificity of 100\%, and accuracy of 93\% for the detection of viable tumor after TACE (in $30 \mathrm{HCCs}$ ) using double contrast MRI [superparamagnetic iron oxide (SPIO) and Gd-DTPA agents].

Subtraction gained higher sensitivity and specificity and confidence interval by:

- Providing specific contrast enhancement patterns. 
- Peripheral nodular enhancement for residual disease.

- Rim contrast enhancement for reactive perilesional hyperaemia.

- No enhancement for well ablated lesions

Limitations of our study are that it was a retrospective study so it is prone to the limitations inherent to all studies of this design. The sample size was small due to the strict selection criteria adopted for this highly specialized indication. This reduced the power of the statistical analysis. The Standard of reference SOR was not based on histology. No biopsies were performed to confirm complete treatment necrosis or residual disease, due to ethical and practical difficulties in performing transional zone (TZ) biopsies in all cases.

\section{Conclusion}

Finally we conclude that, MRI is a powerful tool in detection of tumor viability and complications after TACE. Imaging protocol should include dynamic study combined with post processing subtraction images for better tissue characterization. DWI can be used for patients who can't receive GDDTPA and to quantify tumor necrosis in large HCC. Compared to DWI, Subtraction MRI is much more valuable, where it increases radiologists' confidence in interpreting treatment response following loco-regional therapies for HCC.

\section{References}

1. Llovet JM, Pavel M, Rimola J, Diaz MA, Colmenero J, Saavedra-Perez D, et al. Pilot study of living donor liver transplantation for patients with hepatocellular carcinoma exceeding Milan Criteria (Barcelona Clinic Liver Cancer extended criteria). Liver Transplant. 2018;24(3):369-79.

2. Sugawara Y, Yamamoto H, Hibi T. Living donor liver transplantation for patients with hepatocellular carcinoma in Japan. Hepatoma Res. 2018;4:33.

3. Ferri J, Dockx Y, Vonghia L, Papadimitriou K, Rasschaert M, Francque S, et al. Innovative molecular targeted agents in hepatocellular carcinoma: new gladiators on the arena. Minerva Chir. 2017;72(3):206-18.

4. Liu B, Jiang C, Chen S, Huang G, Wang Y, Li $\mathrm{J}$, et al. Multipronged ethanol ablation combined with TACE for intermediate hepatocellular carcinoma. Minim Invasive Ther Allied Technol. 2018;27(5):300-8.

5. Bellissimo F, Pinzone MR, Cacopardo B, Nunnari G. Diagnostic and therapeutic management of hepatocellular carcinoma. World J Gastroenterol. 2015;21(42):12003.

6. Kierans AS, Elazzazi M, Braga L, Leonardou P, Gerber DA, Burke C, et al. Thermoablative treatments for malignant liver lesions: 10-year experience of MRI appearances of treatment response. Am J Roentgenol. 2010;194(2):5239.

7. Newatia A, Khatri G, Friedman B, Hines J. 
Subtraction imaging: applications for nonvascular abdominal MRI. Am J Roentgenol. 2007;188(4):1018-25.

8. Rimola J, Davenport MS, Liu PS, Brown T, Marrero JA, McKenna BJ, et al. Diagnostic accuracy of MRI with extracellular vs. hepatobiliary contrast material for detection of residual hepatocellular carcinoma after locoregional treatment. Abdom Radiol. 2019;44(2):549-58.

9. Bolog $\mathrm{N}$, Pfammatter $\mathrm{T}$, Müllhaupt $\mathrm{B}$, Andreisek G, Weishaupt D. Double-contrast magnetic resonance imaging of hepatocellular carcinoma after transarterial chemoembolization. Abdom Imaging. 2008;33(3):313-23.
10. Goshima S, Kanematsu M, Noda Y, Kondo H, Watanabe H, Bae KT. Diffusion kurtosis imaging to assess response to treatment in hypervascular hepatocellular carcinoma. Am J Roentgenol. 2015;204(5):W543-9.

11. Choi S-Y, Kim SH, Park CK, Min JH, Lee JE, Choi Y-H, et al. Imaging Features of Gadoxetic Acid-enhanced and Diffusionweighted MR Imaging for Identifying Cytokeratin 19-positive Hepatocellular Carcinoma: A Retrospective Observational Study. Radiology. 2018;286(3):897-908.

12. Akhlaghpoor S, Torkian $\mathrm{P}$, Golzarian J. Transarterial bleomycin-lipiodol embolization (B/LE) for symptomatic giant hepatic hemangioma. Cardiovasc Intervent Radiol. 2018;41(11):1674-82.

To cite this article: Medhat M. Reffat, Wael Elshawaf, Shorouk Z. Abdel Shafy, Khaled E. Aly. The Role of Dynamic Subtraction MRI in assessment of reactivity of HFLs post TACE by 3 tesla MRI machine. BMFJ 2021;38(1): 344-352 DOI: 10.21608/bmfj.2021.50243.1345 